\title{
The Response of Magnetic Instruments to Earthquake waves
}

\author{
Folke ELEMAN* \\ Swedish Board of Shipping and Navigation, Stockholm 27, Sweden
}

(Received Aug. 15, 1965)

\begin{abstract}
Part I. The response of standard magnetographs to earthquake waves is a seismograph effect. These instruments react as ultra-low-sensitivity seismic recorders. With little or no additional effort some magnetic observatories could be used as supplementary seismic stations at the times of great earthquakes. The effects of the Alaskan earthquake of March 28,1964, are studied in detail in this paper. A number of similar cases are known.

Part II. Even if the response of standard magnetographs is definitely of a mechanical nature it is likely that the earthquake waves do generate real magnetic waves. Some experimental evidence (from Bergen Park, Colorado) of the existence of such waves is presented. Mechanisms for generation of the observed waves are discussed. It is shown that piezomagnetic oscillations in magnetic rock or induced currents in a region with enhanced conductivity may offer an explanation. These effects are associated with anomalous conditions in the crust. It seems evident that the properties of the "average crust" cannot explain the observed magnetic variations.
\end{abstract}

\section{Introduction}

Since the early days of continuous photographic recording of the geomagnetic field the response of magnetographs using suspended magnets to earthquakes has attracted the interest of many observers. Opinions have differed regarding the "reality" of these effects. Some have ascribed them to electric or magnetic oscillations generated by the earthquake, others to mechanical vibrations of the magnetic instruments.

Davison (1905) reported on thirteen magnetic observatories recording disturbances from the Riviera earthquake of February 23, 1887.

While Bauer was in charge of the geomagnetic work of the U.S. Coast and Geodetic Survey he arranged for the installation of primitive seismographs at four magnetic observatories. He thus provided means for direct comparison of the two types of earthquake record. The results were discussed by Burbank (1905) and Bauer (1906). These investigations showed that some earthquakes were recorded on the magnetographs but not on the seismographs, and vice versa. The onset times of the magnetic disturbances generally agreed roughly with the times of arrival of the seismic waves, when both kinds of waves were observed.

Mascart (1887) believed that the variations recorded by the magnetograph were real, i.e. caused by variations in the magnetic field rather than by mechanical vibrations. $\mathrm{He}$ tried to explain them by means of electric currents generated by the earthquake.

Moureaux and Mascart (1889) attempted to decide between the mechanical and magnetic theory by using a second variometer where the suspended magnet had been replaced by a

* Now at Goddard Space Flight Center, Maryland, U.S.A. 
copper bar. At the time of subsequent earthquakes the magnet oscillated but the copper bar did not. This result was thought to preclude a mechanical explanation of the phenomenon.

The response considered as a mechanical effect was discussed tentatively by Davison (1885). He confined himself to compass-needles. The case of suspended systems (unifilar and bifilar) was attacked mathematically by Liznar (1895) and, independently, by Reid (1914). A summary of their argumentation will be given later.

Chapman (1930) reported two incidents when ships' compasses were acting queerly in connection with earthquakes. The observed variations appear to have been to one side rather than of oscillatory type. Since the ships were moving, the explanation might be that they passed over spatial anomalies which have nothing to do with the earthquakes. Without checking this the observations are inconclusive.

The present writer proposes an explanation of the instrumental response to seismic waves which differs somewhat from that of the earlier workers. It is shown that a magnetic torque is introduced by the pendulum swing of the suspended magnets. The magnetographs may in fact in some cases be used as quantitative seismographs of ultra-low sensitivity.

Even if the response of standard magnetographs is definitely of a mechanical nature, it is likely that the earthquake waves do generate real magnetic waves. The expected amplitudes of these are calculated for three simple models, the piezomagnetic model, the field gradient model and, the induction model. It is found that these effects are usually too small to be observed against the background of geomagnetic noise. There may however be exceptional cases when "seismomagnetic waves" can actually be observed. Some experimental evidence (from Bergen Park, Colorado) of the existence of such waves is presented.

\section{Part I, Seismograph effect}

\section{Observed distubances}

Fig. 1 shows typical variations follwing a great earthquake, in this case the Alaskan earthquake of March 28, 1964. They were obtained at the Swedish magnetic stations, Kiruna and Enköping. The primary field sensors of these instruments (modified Grenet pulsation recorders) are suspended magnets. This is a common feature of most of the magnetic stan-

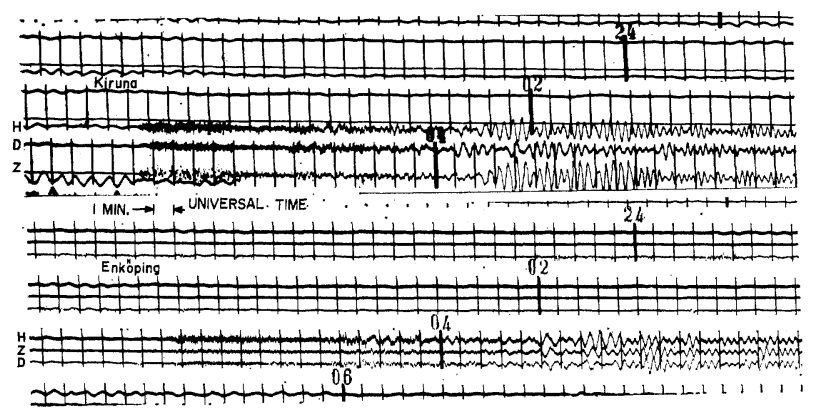

Fig. 1 Earthquake vibrations rezorded at Enköping and Kiruna in Sweden (modified Grenet pulsation recorders), March 28, 1964. 
dard instruments around the world. The Alaskan earthquake has been recorded in a similar way at a large number of the geomagnetic observatories.

The sensitivity of the Kiruna and Enköping magnetographs is unusually high for instruments with suspended magnet. This is due to special construction features; see Voelker (1963). The vertical lines in Fig. 1 indicate minutes and hours. The sensitivity of the records depends on the period of the variations. For a given period it is however nearly the same at both stations for any one component. The largest amplitude seen is that for Kiruna $Z$ at about $04 \mathrm{~h} 04 \mathrm{~m}$ (U.T.). It is about $3 \gamma$. It should be noted that the disturbances start with an oscillation of short period $(2-4 \mathrm{sec})$, followed by an oscillation of longer period (12-30 sec).

The basic data for the Alaskan earthquake are as follows: Origin time: March 28, 1964, 03h36m10s U.T. Epicenter: $61.1^{\circ} \mathrm{N}, 147.8^{\circ} \mathrm{W}$. Depth: about $20 \mathrm{~km}$. Magnitude: 8.5 .

The starting times of the magnetic effects as found on the records are: for Kiruna $03 \mathrm{~h}$ $45 \mathrm{~m} 12 \mathrm{~s}$, for Enköping 03h46m18s. These times have been compared with the times of arrival of the seismic $p$ waves at the adjacent seismic stations at Kiruna and Uppsala; see Båth (1965). With due corrections for differences in position we find that the times of the magnetic and the seismic disturbances agree within a few seconds. This applies also to a number of details in the two kinds of records, e.g. the occurrence of long periodic waves more than 15 minutes after the start of the first oscillations and the phase relations between the horizontal and vertical components of the Rayleigh waves. Similar results have been confirmed by several other observatories.

Fig. 2 shows an example of the same disturbance as recorded on the standard rapidrun magnetograph at Tucson, Arizona (U.S. Coast and Geodetic Survey).

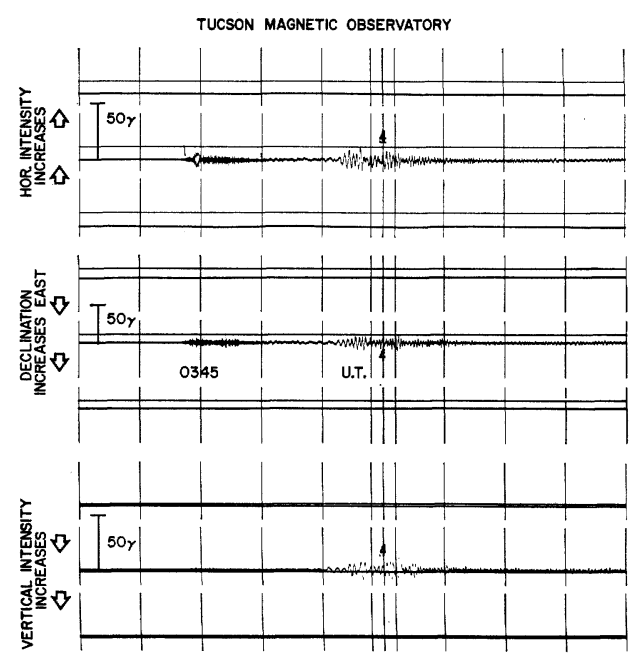

Fig. 2 Earthquake vibrations recorded at Tucson, Arizona, March 28, 1964. 


\section{Nature of response}

In order to get an independent check of the magnetic effects described above, magnetic records derived from other types of equipment were inspected. Induction magnetometers with coil sensors are operated in Kiruna (U.S. Bureau of Standards type) and Uppsala (Benioff type). Neither of these showed any magnetic effect. On the contrary, they indicated unusually quiet conditions. Only one explanation of this seems tenable: the magnetic standard equipment with suspended magnets responds as a seismograph to this kind of disturbance.

This result is not so obvious as might be supposed. Except at locations which are close to the epicenter, he earth's movements are virtually parallel translations. Recorded magnetic variations imply a rotary motion of the suspended magnet. It is not immediately clear how the earthquake tremor could produce such a motion.

\section{The response of $H$ and $D$ variometers}

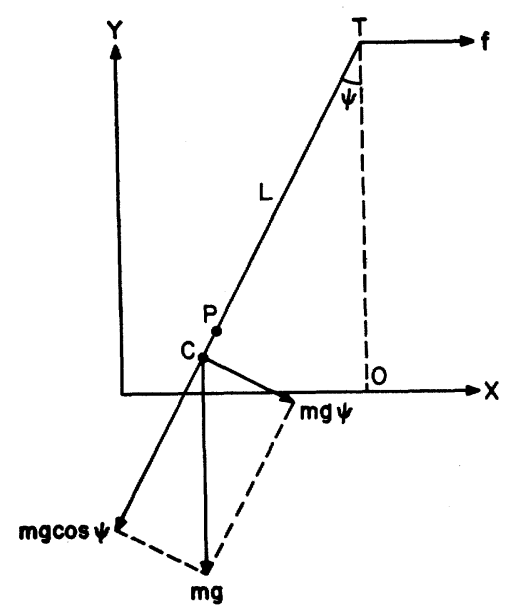

Fig. 3 The magnetic system of the $D$ variometer as seen from magnetic south.

Fig. 3 illustrates schematically the performance of the suspended magnet of a $D$ (declination) variometer being subject to a horizontal vibration. For simplicity, we confine ourselves to a sinusoidal motion of amplitude $a$ perpendicular to the local magnetic meridian. (Extension to a more general motion and to the $H$ variometer can easily be made).

The earth tremor makes itself felt as vibrations of the torsion head $T$ where the fiber is attached. It causes the magnet to deviate from its unperturbed position $O$ and start pendular oscillations. The magnet has the mass $m$; $L$ is the length of the fiber, or rather the distance between $T$ and the center of mass $(C)$ of the magnet system (including mirror etc.).

The $Y$-axis represents the undisturbed position of the system at the time $t=0$ when the tremor begins. A horizontal force $f$ then starts to act on the upper end of the fiber. At the time $t$ the magnet (C) has reached a position $(x ; y) . T$ has then the position $(\xi ; L)$. The difference in horizontal displacement between $T$ and $O$ is denoted by $x^{\prime}=\xi-x$. For the following short time interval $d t$ we have the energy equation

$$
f\left(d x+d x^{\prime}\right)=m \frac{d^{2} x}{d t^{2}} d x+m\left(\frac{d^{2} y}{d t^{2}}+g\right) d y
$$

Assuming a slow motion we get $\quad f=m \frac{d^{2} x}{d t^{2}}$ and

$$
f d x^{\prime}=m g d y
$$

The last two equations give for small oscillations 
\[ \begin{array}{c}f=m g \frac{d y}{d x^{\prime}}=m g \frac{x^{\prime}}{L} \\ \text { and } \quad \frac{d^{2} x}{d t^{2}}=g \frac{x^{\prime}}{L}\end{array} \]

If we assume a horizontal displacement in the magnetic East-West direction of the form

we get

$$
\begin{gathered}
\xi=\dot{a} \sin \omega t \\
\frac{d^{2} \xi}{d t^{2}}=\frac{d^{2} x}{d t^{2}}+\frac{d^{2} x^{\prime}}{d t^{2}}=-\omega^{2} a \sin \omega t
\end{gathered}
$$

Inserting this in (2) we get

$$
\frac{d^{2} x^{\prime}}{d t^{2}}+g \frac{x^{\prime}}{L}+\omega^{2} a \sin \omega t=0
$$

In deriving this equation for the pendulum swing of the magnet we have neglected the damping and the rotation of the magnet. This is justified for the small, slow variations we are studying here. For a more general approach, see Appendix I.

Equation (4) can be written

$$
\frac{d^{2} \Psi}{d t^{2}}+\omega_{p}^{2} \Psi+\frac{\omega^{2} a}{L} \sin \omega t=0
$$

where $\omega_{p}$ is the natural pendulum frequency of the magnet system.

The solution contains a natural vibration term. This dies out due to the damping which although small, is of course always present (air friction, eddy currents, etc.). We also get a steady forced vibration

$$
\Psi=-\frac{a \omega^{2}}{L\left(\omega_{p}^{2}-\omega^{2}\right)} \sin (\omega t-\delta)
$$

where $\delta$ is the phase angle. For $\omega_{p}^{2} \gg \omega^{2}$

we get the amplitude

Since

$$
\Psi_{0}=\frac{a \omega^{2}}{L \omega_{p}^{2}}=\frac{a T_{p}^{2}}{L T^{2}}
$$

$$
T_{p}=2 \pi \sqrt{\frac{L}{g}}
$$

this can be written

$$
\Psi_{0}=\frac{4 \pi^{2} a}{g T^{2}}
$$

It is obvious that, due to the pendulum swing, the geomagnetic $Z$ field will have a component $Z \Psi$ whih is perpendicular to the plane through the magnet and its axis of rotation, defined by the fiber. $Z \Psi$ introduces a torque which for small values of $\omega$, brings the magnet to turn until it reaches an equilibrium angle $\theta$ defined by $H \theta=Z \Psi$ where $H$ is the horizontal field. (We are here neglecting the torsion of the fiber).

The magnetographs will record an apparent magnetic vibration in the $D$ component 
(regardless of sign and phase)

$$
\Delta D=\frac{4 \pi^{2} Z a}{g T^{2}} \sin \omega t
$$

For our purpose we can with sufficient accuracy use $g=980 \mathrm{~cm} \mathrm{sec}^{-2}$ for the whole earth. Then (6) may be written

$$
\Delta D_{0}=\frac{\Delta D}{\sin \omega t}=4.0 \times 10^{-2} \frac{Z a}{T^{2}}
$$

It is easy to show (see Appendix 2) that we will get exactly the same equation for $\Delta H$ if $a$ signifies the amplitude of an earth tremor in the direction of the local magnetic meridian. The results also apply to $X$ and $Y$ variometers.

It is thus seen that the recorded magnetic trace will show a vibration with the same period as the earthquake waves. Its amplitude is determined by the period and the amplitude of the earth movements, and the local magnetic field. For actual evaluation the magnetic scale value must also be known.

Equation (6) is valid as long as $T$ is long compared with the natural periods of the pendulum $\left(T_{p}\right)$ and of the magnet $\left(T_{M}\right)$. This condition is generally fullfilled for the long periodic seismic surface waves. For the short periodic waves the expressions get somewhat more complicated. See Appendix 1.

\section{Reid's approach}

Reid (1914) has also attacked the problem of the response of suspended magnets to earth vibrations. He has somewhat extended an earlier investigation by Liznar (1895). An outline of Reid's argument will be given here.

For the system at rest the point of support $(P)$ where the fiber is attached to the magnet system is generally not in the same vertical line as the center of mass $(C)$ of the magnet system. When the system performs pendular oscillations the tension of the fiber may be resolved in a vertical and a horizontal part. Reid assumes that the latter part will tend to turn the magnet about $C$. Since the tension equals the weight of the magnet system the torque would be (leaving out second order effects)

$$
m g \Psi c
$$

where $c$ is the (horizontal) distance between $P$ and $C$.

Reid further assumes that the distance $c$ can be determined by equating the opposing torques from the gravitation and the magnetic $Z$ field. When the magnet is horizontal this gives

$$
c=\frac{Z M}{m g}
$$

where $M$ is the magnetic moment.

On the basis of the prevous assumptions Reid derives the formulas for free oscillations (damped and undamped) and also for forced oscillations due to earth tremor. He restricts 
himself to the cases when the periods of the earthquake waves closely agree with the period of the pendular or the magnetic oscillation.

It is doubtful, however, if the reasoning of Liznar and Reid is tenable in the case of a suspended magnet. The only force, except $Z$, having a component perpendicular to the plane through the magnetic axis and the axis of rotation (assumed to coincide with the fiber) and thus capable of producing a rotation, is the gravity force $m g$. But since $m g$ acts at the center of gravity no rotation occurs. Also, the expression for $c$ overlooks the stiffness of the (quartz) fiber which is probably important.

Solving Reid's equations for the long periodic case (cf Section 5) we once more get the result (6), in spite of the different mechanisms assumed. If both mechanims were effective simultaneously they would in fact give twice the effect (6). As will be seen in later sections most observations seem to agree better with the deviation $\Delta D$ than with $2 \Delta D$. Since the magnetic torque mechanism introduced in this paper appears to be an inevitable consequence of the pendulum swings it is probably the only effective mechanism.

It is possible to conceive such deviations from the types of oscillations assumed here that Reid's approach would be at least partly applicable. One such deviation is that the axis of rotation does not coincide with that of the fiber. However, this alternative seems unlikely for the small oscillations considered here.

It has sometimes been thought that the response of $H$ and $D$ variometers to earth tremors can be explained by their structural similarities to the torsion seismometer of Anderson and Wood (1924). However, in that case the weight is suspended from above and below. The weight does therefore not perform the kind of pendulum swing which is essential for the explanation given here (Section 3).

As pointed out by Reid the $H$ and $D$ variometers would be sensitive to vertical earthmotions of period $T$ only if $T_{p}$ is commensurate with $T$. Thus we need not consider this effect here.

\section{The compass needle}

The expression (9) is exactly valid in the case of a compass needle pivoting about a vertical pin. It is therefore of a certain interest to study the apparent magnetic variation due to (9).

The torque due to the earth tremor causes the magnet to seek a new position of equilibrium defined by

$$
m \frac{d^{2} \xi}{d t^{2}} c=M H \theta
$$

Here we have assumed that $c\left(d^{2} \theta / d t^{2}\right) \ll d^{2} \xi / d t^{2}$ and $T_{M} \ll T$. H $\theta$ is equivalent to an $E W$ disturbance $\Delta D$. Inserting (3) and (9) in this expression we get

$$
\Delta D=\frac{4 \pi^{2} Z a}{g T^{2}} \sin \omega t
$$

This is the expression (6). Thus a suspended magnet and a compass needle respond in 
the same way. The result also indicates why Reid's approach gives the same response.

\section{Numerical values}

For $Z=0.47$ gauss (Stockholm area) equation (7) yields

$$
\Delta D_{0}=1.88 \times 10^{3} \frac{a}{T^{2}} \gamma
$$

According to Båth (1965) the $E$ component of the $M$ waves recorded at Uppsala after the Alaskan earthquake had the amplitude $a=0.226 \mathrm{~cm}$ and the period $T=22 \mathrm{sec}$. Inserting this in (10) gives $\Delta D=0.88 \gamma$.

The actual maximum values of $\Delta D_{0}$ observed at the two adjacent magnetic observatories Lovö and Enköping are respectively, $0.8 \gamma$ and $0.9 \gamma$. The period is $22 \mathrm{sec}$. The agreement is good considering that the recorded magnetic variations have amplitudes of only about $1 \mathrm{~mm}$ or less.

Turning to the $H$ component at Lovö we find that the amplitude of the simultaneous $H$ variations is $1.0 \gamma$. The corresponding period is about $22 \mathrm{sec}$. According to (10) this gives a displacement amplitude in the NS direction

$$
a_{N}=\frac{\Delta H_{0} T^{2}}{1.88 \times 10^{3}}=0.26 \mathrm{~cm}
$$

Considering the geographical position of the station this would roughly agree with the amplitude of the horizontal component of the Rayleigh waves. The corresponding vertical component would be about $0.44 \mathrm{~cm}$, assuming the ratio 1.7 between the vertical and the horizontal displacements.

\section{7. $\mathrm{Z}$ variometer}

The conventional $Z$ variometer consists of a horizontal magnet rotating about a horizontal axis defined by knife edges or fibers. In this case the response to tremors depends on the separation between the pivot axis and the center of mass.

This separation can be resolved into a part $c$ parallel to the magnetic axis, and a part

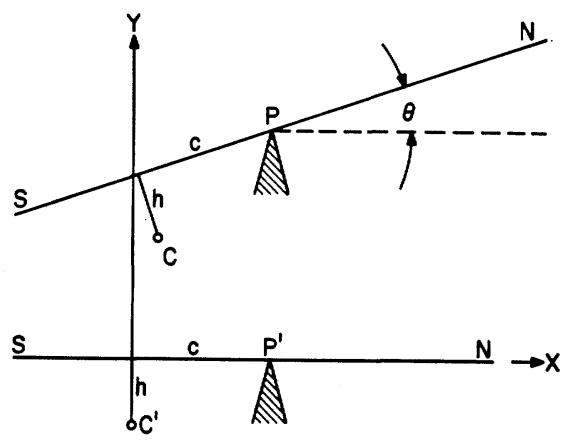

Fig. 4 A $Z$ magnet in $E W$ direction, seen from the south, in two different tremor positions. 
$h$ perpendicular to the axis (see Fig. 4). The former part is rigorously determined by (9). The part $h$ decides the sensitivity of the variometer. For $h=0$ the magnet become unstable if directed in magnetic $E W$.

Fig. 4 pictures an $E \mathrm{~W}$-oriented $Z$ magnet (of mass $m$ and moment of inertia $I$ ) in two positions. Due to an earth-tremor the pivot-point $P$ is displaced vertically a distance $\xi$. The center of mass $C$ moves the distance $y$ and the magnet turns a small angle $\theta$. This gives the connection

$$
\xi=y+c \theta=a \sin \omega t
$$

If $f$ is the vertical force acting at $P$ we get (neglecting damping)

$$
f(d y+c d \theta)=m \frac{d^{2} y}{d t^{2}} d y+m g h \theta d \theta+I \frac{d^{2} \theta}{d t^{2}} d \theta
$$

After separation of translational and rotational terms these expressions give

$$
I \frac{d^{2} \theta}{d t^{2}}+m g h \theta=-m c a \omega^{2} \sin \omega t
$$

The stationary solution is for small values (regardless of sign and phase)

$$
\theta=\frac{m c a}{I} \frac{T_{M}^{2}}{T^{2}} \sin \omega t
$$

where

$$
T_{M}^{2}=4 \pi^{2} \frac{I}{m g h}
$$

It may be shown that a horizontal earth tremor $a \sin \omega t$ along the axis of the magnet would give the same expressions for $\theta$ but with $c$ replaced by $h$.

The discussion can be extended to the case of a $Z$ magnet of arbitrary orientation. This is done by adding the term $M H \theta \cos \alpha$ to the left side of the difierential equation ( $\alpha=$ magnetic azimuth). The result (11) remains formally the same, with

$$
T_{M}^{2}=4 \pi^{2} \frac{I}{m g h+M H \cos \alpha}
$$

For a qualitative discussion of the performance of the $Z$ balance under resonance conditions, see Reid (1914).

\section{Numerical chech of $Z$}

The apparent magnetic deviation corresponding to the vertical motion is

$$
\Delta Z_{v}=S_{z} \theta
$$

where $S_{Z}$ is the magnetic scale-value defined by

$$
S_{z}=\frac{m g h+M H \cos \alpha}{M} \frac{\text { gauss }}{\text { radian }}
$$

The expressions (9), (11), (12), (13) and (14) now yield

$$
\Delta Z_{v}=\frac{4 \pi^{2} a Z}{g T^{2}} \sin \omega t
$$


This is exactly the same expression as that derived for the $D$ and $H$ magnetometers and the magnetic compass. For a horizontal earth motion in the direction of the magnet we get

$$
\Delta Z_{h}=\frac{h}{c} \frac{4 \pi^{2} a Z}{g t^{2}} \sin \omega t
$$

When-as is often the case $-h \ll c, \Delta Z_{h}$ may be neglected in comparison with $\Delta Z_{v}$. Otherwise, the $Z$ trace displays a combined vertical and horizontal motion.

At the Lovö Observatory ( $Z=0.47$ gauss) the ratio $h: c$ is roughly 0.25 . The following values were observed at one phase of the earthquake:

$$
\Delta Z=1.4 \gamma, \quad T=22 \mathrm{sec} .
$$

Inserting in (15) we get

$$
a=0.36 \mathrm{~cm}
$$

This is to be compared with the value $0.44 \mathrm{~cm}$ obtained in Section 6 as a rough estimate from the observed horizontal amplitude of the Rayleigh waves.

\section{Magnetographs as supplementary seismographs}

When looking for world wide seismic data associated with the Alaskan earthquake of March 28, 1964, the present writer could not avoid observing the great number of cases when the seismographs (short periodic and long periodic) failed to record properly the large surface waves studied here. Either the seismograms displayed a confusion of traces impossible to disentangle, or the traces disappeared altogether.

In most cases use has therefore been made of the nomogram for the maximum ground amplitude as a function of earthquake magnitude and distance from source, compiled by Gutenberg and Richter (1956). It pertains to the combined horizontal components for surface waves of 20 -second period.

The general impression is that for earthquakes like the Alaskan event a net of ultralow-sensitivity seismographs would be needed. The existing strong-motion instruments (like those on the American west coast) do not provide a satisfactory coverage.

In this situation the rapid-run standard recorders of the geomagnetic observatories may in some cases be useful as a supplement to the seismic network. The magnetographs should be of value within a range of about $30^{\circ}-80^{\circ}$ from the source. As may be seen from the response equations derived for long-periodic seismic waves they can be made quantitative through the determination of a few instrumental constants (most of which have to be known

anyhow). With some additional work (including determination of the damping constant) a "magnification curve" could be derived for periods down to about $5 \mathrm{sec}$. The time resolution of the magnetic records is, however, generally not sufficient for studies of the short periodic waves.

Evidently, the magnetic records could be used for this purpose only at times when the magnetic conditions are reasonably quiet. The Alaskan earthquake for example occurred in a very quiet period. Especially in years of sunspot minima such events would not be rare. 
The method should have unrestricted application to the horizontal wave components. $Z$-records could be used for evaluation of vertical movements when $c \gg h$, which is often the case (an important exception being when the north seeking end of the magnet points in a southerly direction).

The phase angles (neglected in the previous discussion) are easily determined from the instrument constants. In the case $T \gg T_{p}$ and $T \gg T_{m}$ we have $\delta=0$ for all components. The relation between the direction of the earth movement and the recorded deviation has to be established for each individual instrument.

Since the amplitudes of the seismic $\Delta D, \Delta H$, and $\Delta Z$ are proportional to $Z$, the observations would be confined to those portions of the earth where $Z$ is sufficiently large. The position of the border-lines is determined by the scale-value of the instruments. In Huancayo where $Z$ is only about $1000 \gamma$, no seismic effect from the Alaskan earthquake could be observed. The combined horizontal ground amplitude was about $2.3 \mathrm{~mm}$ and would have been observed at stations on medium high north or south latitudes. Questions such as the positions of the observatories, ground conditions etc., must of course also be contemplated. A list of observatories operating magnetic recorders suitable as supplementary seismographs could perhaps be compiled through appropriate bodies within the IUGG.

The instrument theory presented above specifically pertains to conventional variometers. It is probably also applicable to pulsation recorders of the Grenet-Voelker type (Fig. 1). In these the primary field sensors are magnets, the rotation of which induces currents in a surrounding coil. Although the pure pendulum swing of the $H$ or $D$ magnets would produce currents, the rotation is probably the main contributor. This is confirmed, at least for periods above $20 \mathrm{sec}$, by comparison with adjacent LaCour instruments. A chain of pulsation recorders has recently been established in Europe.

\section{Suggested experiments}

For an experimental control of the response of magnetic instruments to vibrations, a movable support would be needed. It should perform smooth sinusoidal movements (horizontal or vertical) with an amplitude of, say, $1 \mathrm{~cm}$ and periods approximately within the range of 10-50 sec. The performance of the magnet could be studied either by means of a split photocell recorder, or simply by observations with an autocollimator mounted on the instrument.

For horizontal variometers it would probably be sufficient to make calibrations for each type of suspended system (Ruska, LaCour, etc.), the variations being small.

By making the experiment inside a Helmholtz coil the effect of $Z$ on the response of the horizontal variometers could be checked.

Deviations from the theoretical amplitude formulae might be expected in cases when the lateral rigidity of the fiber is important in comparison with the mass of the magnet. The likelihood of this should generally be greater for $H$ than for $D$ variometers.

Comparisons between magnetograms and seismograms (Lovö-Uppsala) indicate that the differences between theoretical and experimental response might be small, in many 
cases probably insignificant.

Since the observed vibrations are due to a magnetic torque, the result of the experiment of Moureaux and Mascart (mentioned in the "Introduction") is to be expected. It supports the theory presented in this paper.

\section{Worldwide observations}

Table 1 lists the observed maximum horizontal displacements for the Alaskan event as derived from the magnetic records of a number of observatories. The observatories are arranged according to distance from the epicenter $\left(61.1^{\circ} \mathrm{N}, 147.8^{\circ} \mathrm{W}\right)$.

Table 1

\begin{tabular}{l|r|r|c|c|c|c|c|c|c}
\hline \multicolumn{1}{c|}{ Station } & Lat. & Long. & $\begin{array}{c}\text { Time } \\
\text { U.T. }\end{array}$ & $\begin{array}{c}\text { Dist. } \\
\text { degrees }\end{array}$ & $\begin{array}{c}\text { Seism. } \\
\Delta H \mathrm{~mm}\end{array}$ & $\begin{array}{c}T_{H} \\
\text { sec. }\end{array}$ & $\begin{array}{c}\text { Seism. } \\
\Delta D \mathrm{~mm}\end{array}$ & $\begin{array}{c}T_{D} \\
\text { sec. }\end{array}$ & $\begin{array}{c}\text { Nomo- } \\
\text { gram }\end{array}$ \\
\hline Tucson & $32^{\circ} 15^{\prime}$ & $-110^{\circ} 50^{\prime}$ & 0358 & 39 & 14.8 & 19 & 11.3 & 18 & 12.0 \\
Honolulu & $21^{\circ} 19^{\prime}$ & $-158^{\circ} 00^{\prime}$ & 0357 & 42 & 6.7 & 10 & 7.0 & 10 & 9.0 \\
Leirvogur & $64^{\circ} 11^{\prime}$ & $-21^{\circ} 42^{\prime}$ & 0406 & 48 & 8.1 & 20 & 5.8 & 20 & 7.7 \\
Tromsö & $69^{\circ} 40^{\prime}$ & $18^{\circ} 57^{\prime}$ & 0406 & 49 & 7.0 & 21 & 0.9 & 22 & 5.3 \\
Kiruna & $67^{\circ} 50^{\prime}$ & $20^{\circ} 25^{\prime}$ & 0409 & 51 & 4.2 & 22 & 1.4 & 21 & 5.3 \\
Nurmijärvi & $60^{\circ} 31^{\prime}$ & $24^{\circ} 39^{\prime}$ & 0408 & 59 & 3.0 & $(22)$ & 2.2 & & 5.0 \\
Lovö & $59^{\circ} 21^{\prime}$ & $17^{\circ} 50^{\prime}$ & 0410 & 61 & 2.2 & 22 & 6.1 & 22 & 4.9 \\
Rude Skov & $55^{\circ} 51^{\prime}$ & $12^{\circ} 27^{\prime}$ & 0413 & 62 & 2.4 & 27 & 1.7 & 27 & 4.6 \\
Witteveen & $52^{\circ} 49^{\prime}$ & $6^{\circ} 40^{\prime}$ & 0416 & 64 & 4.4 & 22 & & 20 & 4.2 \\
Dourbes & $50^{\circ} 06^{\prime}$ & $4^{\circ} 36^{\prime}$ & 0416 & 66 & 4.0 & 23 & 1.7 & 23 & 4.0 \\
Guam & $13^{\circ} 35^{\prime}$ & $144^{\circ} 52^{\prime}$ & 0417 & 68 & 2.4 & 17 & & & 4.0 \\
Toledo & $39^{\circ} 53^{\prime}$ & $-4^{\circ} 03^{\prime}$ & 0421 & 76 & 2.8 & 20 & & & 3.3 \\
Port Moresby & $-9^{\circ} 24^{\prime}$ & $147^{\circ} 09^{\prime}$ & 0426 & 86 & & & 2.5 & 22 & 2.7 \\
Moca & $3^{\circ} 21^{\prime}$ & $8^{\circ} 40^{\prime}$ & 0445 & 115 & 0.9 & 20 & & & 1.8 \\
\hline
\end{tabular}

The table gives calculated amplitudes and periods for most of the observatories that supplied microfilm copies of their rapid-run magnetograms to World Data Center A before March 1965. In some cases the quality of the reproduction did not allow quantitative measurements. Magnetograms from stations close to the epicenter, like College, Sitka, and Barrow could not be used for determinations of $M$ amplitudes because they were too disturbed. College recorded minor tremors for several hours after the main earthquake.

The last column shows the combined horizontal amplitudes according to the nomogram of Gutenberg, Richter (1956). The nomogram pertains to periods of $20 \mathrm{sec}$. With the exception of Rude Skov and Honolulu this agrees roughly with the observed periods.

The table shows the general trend of amplitude attenuation with increasing distance from the source. It must be borne in mind that the data are from seismically unstandardized observatories whose detailed conditions are not known to the author. Also, there is a certain arbitrariness in the selection of oscillations to be scaled.

It is well known that some soils and sediments tend to amplify the ground motions. The $Z$ field used to calculate the amplitudes is the "official" annual mean submitted by the observatories. It pertains to the pier(s) of the absolute instruments. Owing to natural field 
gradients within the observatory site and disturbance fields from adjacent magnets (neglecting time variations), this $Z$ field generally does not agree with the actual $Z$ field at the quickrun variometers. The fields from the magnets (e.g. $Z$ magnets, temperature-compensation magnets, sensitivity-control magnets) should be easily calculated from the known moments and positions of the magnets. The required accuracy is only about $50 \gamma$.

In some cases the oscillations may be modified by asymmetric damping or magnetic impurities in parts close to the magnet. However, for the long waves mainly considered here the damping terms are normally so small that they have been neglected altogether. Also, many standard magnetographs operate without metallic damping devices.

\section{Response of other hinds of instruments}

As a result of a world-wide request a number of observatories kindly sent copies of their recordings showing various degrees of response to the seismic waves. In some cases the records were accompanied by valuable comments. To present all the records here would be useless repetition. It will be sufficient to reproduce some results obtained at the Niemegk observatory (lat. $52^{\circ} 04^{\prime} \mathrm{N}$, long. $12^{\circ} 40^{\prime} \mathrm{E}$ ) and kindly supplied by Fanselau, Grafe, Schmidt (Private Communication, 1964).

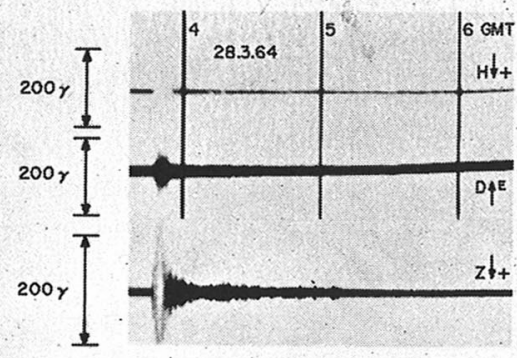

(a)

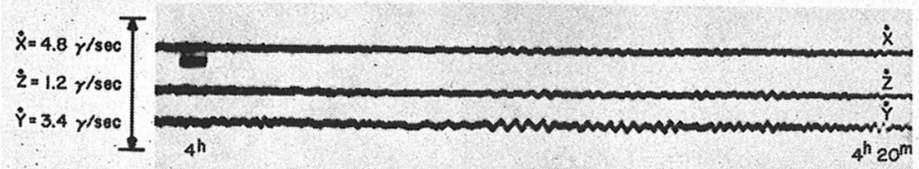

(b)

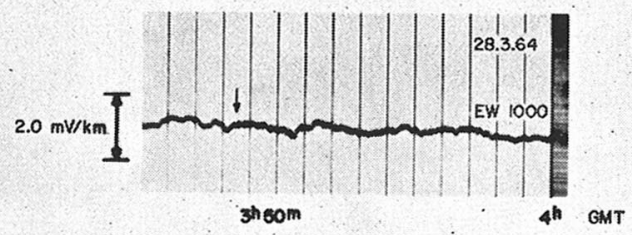

(c)

Fig. 5 Magnetic and electric records from Niemegk, Germany, March 28, 1964. 
Fig. 5a shows the effect on the observatory's insensitive system. This is more or less typical of the appearance on normal-speed magnetographs. The poor time resolution does not permit quantitative measurements.

Fig. $5 \mathrm{~b}$ is the record of an induction magnetometer with three large pick-up coils connected to galvanometers recording photographically. The coils are "air-coils" without cores. It is remarkable that this installation has recorded the earthquake disturbance, with onset time 0347 U.T. It is in all probability a "seismograph effect". It is possible that the galvanometers are responsible for this. If not, the vibrations must have caused some kind of distortion of the coils, changing the magnetic flux. As mentioned before the coil-instruments in Uppsala and Kiruna did not respond to the earthquake.

Fig. $5 \mathrm{c}$ shows the $E W$ component of the Niemegk rapid-run earth-current recorder. Raipid vibrations, hardly discernible in reproduction, start at the time indicated by the arrow. Telluric effects of earthquakes will be discussed in the later part of this paper.

E. Selzer (Private Communication, 1964) reports of observations at the French Observatory Chambon-la-Foret. This observatory operates a 3-component coil magnetometer with mumetal cores ("bar-fluxmeters"). Oscillations appear practically only in the $Z$ component, whereas normally $H$ and $D$ are much more disturbed than $Z$. The $Z$ variations are very similar to the seismic disturbances. Selzer believes that the vertical bar acted as a kind of gravity inclinometer, following the slight inclination of the ground. Another possibility is a mechanical vibration of the pick-up coil.

J.C. Noyes reports similar observations on the record of a coil-core installation operated by Boeing Scientific Research Laboratories, Seattle, Washington. So does J.E. Lokken of Pacific Naval Laboratory, Canada, (Private Communications, 1965).

Even if the magnetic field sensor is insensitive to vibrations the recording equiment (e.g. a pen recorder) may respond. At the Geophysical Institute, College, Alaska, a helium magnetometer was in operation during the earthquake. As may be seen on Fig. 6 it reacted violently to the earth tremor. According to Mather (Private Communication, 1964) the magnetometer recorder acted as poor seismometer. Most of the disturbed part of the record is therefore a seismograph effect. The content of real magnetic effect is probably impossible to determine.

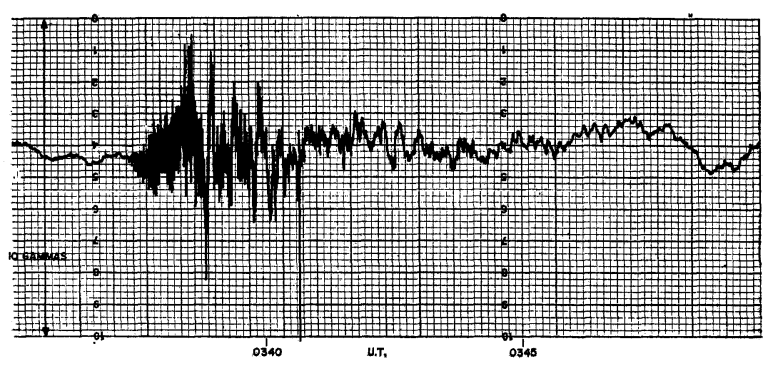

Fig. 6 Magnetic record (Helium Magnetometer) from College Alaska, March 28, 1964. 


\section{Conclusion}

The response of the standard photographic mgnetographs and many other kinds of equipment is a seismograph effect. Any real magnetic variations associated with earthquakes are probably too small to be recorded by the standard instruments. The suspended magnetic system has obvious similarities with the seismic equipment and can in fact be used as an ultra-low-sensitivity seismic recorder. With little or no additional effort some magnetic observatories could operate as supplementary seismic stations at the times of great earthquakes. not coinciding with appreciable magnetic activity. One good example of this is the Alaskan earthquake of March 28, 1964.

\section{Appendix 1.}

A more general derivation of the response expression of the $D$ variometer will be given here.

In the energy equation (1) we may insert one term corresponding to the rotation of the magnet:

\section{$M Z \Psi d \theta$}

where $M$ is the magnetic moment and $d \theta$ is the rotation which occurs while $\Psi$ changes the amount $d \Psi$.

We may further insert the friction term

$$
\alpha \frac{d x^{\prime}}{d t} d x
$$

Note that the friction is proportional to the relative velocity between the magnet and the damping media (air, conductors). The latter move with the velocity $\mathrm{d} \xi / d t$ of the instrument. The velocity dependence is well confirmed experimentally for small velocities.

Assuming an earth movement $\xi=-a \sin \omega t$ this leads to the equation

$$
\frac{d^{2} \Psi}{d t^{2}}+\frac{\alpha}{m} \frac{d \Psi}{d t}+\frac{g}{L}\left(1+\frac{M Z}{m g L} \frac{d \theta}{d \Psi}\right) \Psi=\frac{\omega^{2} a}{L} \sin \omega t
$$

This corresponds to (5).

It can be shown that the expression

$$
\frac{M Z}{m g L} \frac{d \theta}{d \Psi}
$$

is small. According to (9) it may be written (at least approximately)

$$
\frac{c}{L} \frac{d \theta}{d \Psi}
$$

For normal magnets $c$ does not exceed about 0.01 of the length of the magnet. If this is taken to be $1 \mathrm{~cm}$ and if $L=15 \mathrm{~cm}$ we get

$$
\frac{c}{L}=0.0007
$$


The ratio $d \theta / d \Psi$ has in most places the order of magnitude 1 as can be seen from the equilibrium equation

$$
Z \Psi=H \theta
$$

Hence, in all practical cases, the term (16) may be neglected in the parenthesis in the equation above. This may be written

$$
\frac{d^{2} \Psi}{d t^{2}}+\frac{\alpha}{m} \frac{d \Psi}{d t}+\omega_{p}^{2} \Psi=\frac{\omega^{2} a}{L} \sin \omega t
$$

This well-known equation for forced vibrations has for small damping the stationary solution

$$
\Psi=\frac{\omega^{2} a \sin \left(\omega t-\delta_{1}\right)}{L\left[\left(\omega_{p}^{2}-\omega^{2}\right)^{2}+(\alpha / m)^{2} \omega^{2}\right]^{1 / 2}}
$$

The differential equation for the rotation of the magnet is

$$
\frac{d^{2} \theta}{d t^{2}}+\frac{\beta}{I} \frac{d \theta}{d t}+\omega M^{2} \theta=\frac{M Z}{l} \Psi
$$

where $\beta$ is the damping constant.

Inserting the $\Psi$-value from (17) this has the stationary solution

$$
\begin{gathered}
\theta=\frac{M Z \omega^{2} a \sin \left(\omega t-\delta_{1}-\delta_{2}\right)}{I L\left[\left(\omega_{p}^{2}-\omega^{2}\right)^{2}+(\alpha / m)^{2} \omega^{2}\right]^{1 / 2}\left[\left(\omega_{M}{ }^{2}-\omega^{2}\right)^{2}+(\beta / I)^{2} \omega^{2}\right]^{1 / 2}} \\
\operatorname{tg} \delta_{1}=\frac{\alpha \omega}{m\left(\omega_{p}^{2}-\omega^{2}\right)} \\
\operatorname{tg} \delta_{2}=\frac{\beta \omega}{I\left(\omega_{M}{ }^{2}-\omega^{2}\right)}
\end{gathered}
$$

For an ideal bar magnet, the damping factors $\alpha / m$ and $\beta / I$ are equal. In practical variometers this is not the case (mirrors etc.).

For small values of $\omega, \alpha$, and $\beta$ we will once more get the expression (6).

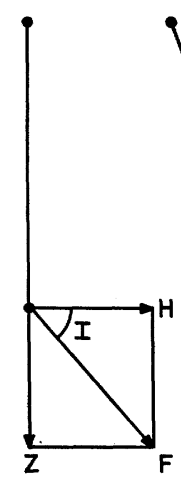

(a)

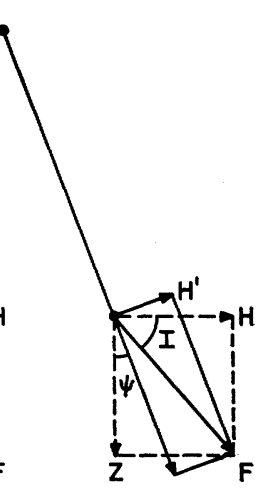

(b)

Fig. 7 The magnetic system of the $\mathrm{H}$ variometer, in two positions, seen from the west.

\section{Appendix 2 .}

Fig. 7 pictures the magnetic system of a $H$ variometer as seen from magnetic East. In the unpertubed position (a) the horizontal field $H$ produces a torque that balances the fiber torsion, thus keeping the magnet in the $E W$ direction.

In (b) the magnet performs a pendulum swing. The magnetic torque is now $M H_{1}$. The change in torque is

$$
M \Delta H=M\left(H-H_{1}\right)
$$

From Fig. 7 we get

$$
\begin{gathered}
\Delta H=H-H_{1}=F[\cos I-\cos (I+\Psi)]= \\
F(\cos I-\cos I \cos \Psi+\sin I \sin \Psi)
\end{gathered}
$$

For small $\Psi$ this gives

$$
\Delta H \approx F \Psi \cdot \sin I=Z \Psi
$$


The result implies that the response of the $H$ variometer is governed by the same expressions as those derived for the $D$ variometer.

\section{Part II. Magnetic effect}

\section{Inertia-free instruments}

Obviously it is not safe to rely on magnetometers with moving parts, galvanometers, pen and ink recorders etc. when tying to determine whether earth tremors produce real magnetic variations.

With the advent of instruments based on nuclear or atomic precession in the last few years the situation has become more hopeful. An inquiry to stations operating such equipment regarding observed effects from the event of March 28, 1964, resulted in a handful of recordings kindly made available by different agencies.

One of the records show magnetic variations that appear to be associated with the earthquake. It is from the U.S. Geological Survey's Geoelectric Observatory at Bergen Park, Colorado. The instruments are housed in the Colorado School of Mines' Cecil H. Green Geophysical Observatory which is designated as "GOL" in the world-wide seismic net. The geographic coordinates are as follows

\section{Lat. $39^{\circ} 42^{\prime} \mathrm{N}$}

Long. $105^{\circ} 22^{\prime} \mathrm{W}$

The variations of the total field intensity are recorded by a meta-stable helium vapor magnetometer, the data being stored on tape. It seems highly improbable that this system in itself is sensitive to vibrations, especially the slow vibrations studied here.

Regarding local sources of disturbance, magnetic bodies performing a relative motion would have an effect. According to W.P. Hasbrouch (Private Communication, 1965) there are no oscillating iron masses (e.g. the pendulum of a seismograph) harmfully close to the sensing head of the magnetometer. Metal structures in the vicinity, e.g. an aluminum roof or a metallic instrument case, would carry induced currents. As shown in a later section their magnetic effect is probably negligible. We can therefore be reasonably confident that the magnetic variations recorded by this equipment reflect a geophysical event having its sources outside man-made installations.

\section{Observed effect}

Fig. 8 shows a playback from the tape recorded at the Bergen Park facility between U.T. $03 \mathrm{~h} 50 \mathrm{~m}$ and $04 \mathrm{~h} 00 \mathrm{~m}$ of March28, 1964. The two upper traces indicate telluric variation, the lower trace the output of the meta-stable helium magnetometer. The labeling in the figure should be sufficient for its present use.

The large variations of the magnetic record stand out very clearly in comparison with the parts of the trace preceding and following the interval covered by Fig. 8 They appear to agree closely in time with the arrival of the large surface waves. Due to difficulties to 


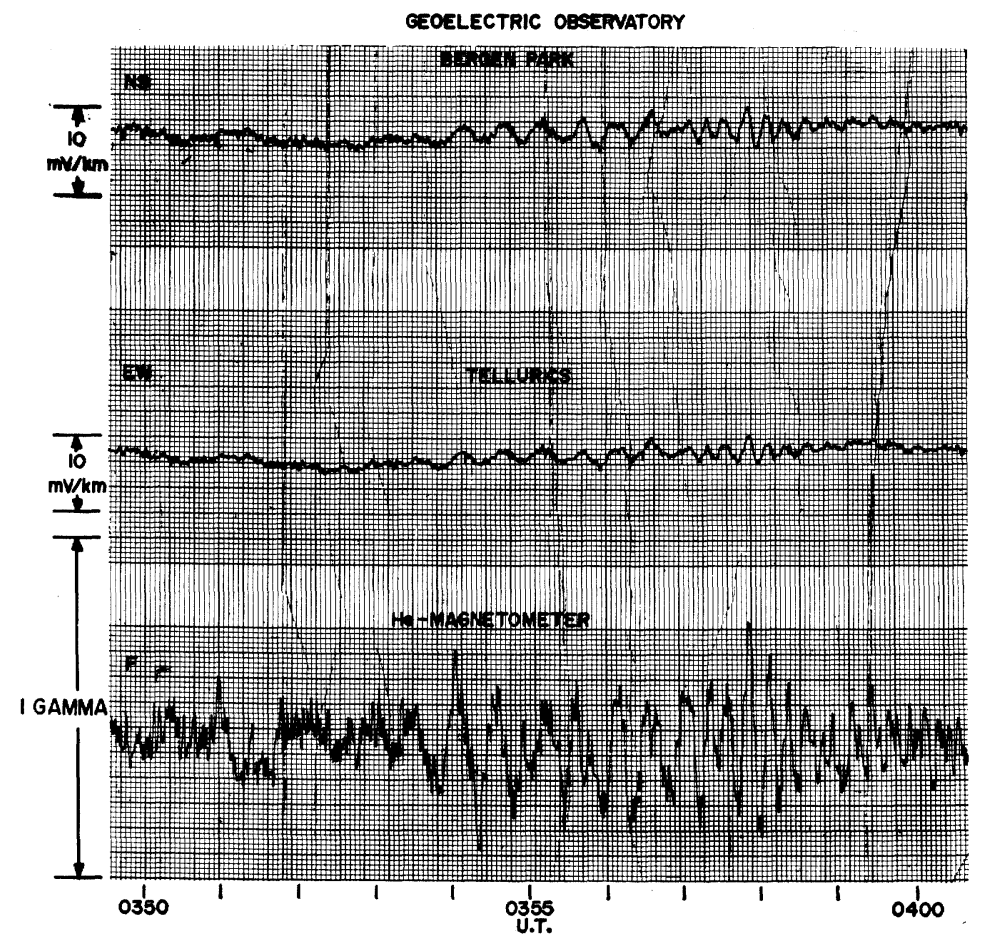

Fig. 8 Magnetic and electric records from Bergen Park, Colorado, March $28,1964$.

interpret the seismograms of the adjacent GOL station this had to be checked in an indirect way, by using the rapid-run magnetograms of the magnetic observatory at Tucson (Lat. $32^{\circ} 15^{\prime} \mathrm{N}$ Long. $110^{\circ} 50^{\prime} \mathrm{W}$ ). These give a very clear picture of the seismic $M$ waves, with a starting time in $Z$ of about $03 \mathrm{~h} 55.5 \mathrm{~m}$; see Fig. 2. When the difference in distance from the focus was corrected (the time difference is about $1.8 \mathrm{~min}$ ) we get a starting time in Bergen Park of $03 \mathrm{~h} 53.7 \mathrm{~m}$. This closely agrees with what is actually observed. As a further check, the periods of the waves (divided in three sub-groups) were determined and compared with the corresponding waves at Tucson (the $Z$ component). The periods of the three groups, 33, 27, and $15 \mathrm{sec}$. agree at the two stations. They also agree with periods of the telluric waves in Fig. 8. These comparisons indicate that the telluric and magnetic variations observed at Bergen Park are associated with the seismic $M$ waves.

It is worth mentioning that the short period $P$ and $S$ waves arriving at $03 \mathrm{~h} 42.8 \mathrm{~m}$ (according to the GOL-seismograms), gave no appreciable effects on the Bergen Park records. The standard magnetographs of the magnetic observatories responded strongly also to these waves. The conclusion is that appreciable magnetic oscillations are only associated with the large surface waves.

\section{Absence of effects.}

The magnetic effect appears to be geographically confined. Four other American sta- 
tions operting Rubidium vapor magnetometers (based on the same principles as the Hemagnetometer) did not record appreciable earthquake effects. The implication of this result will be discussed in a later section.

Unfortunately, no instruments of the inertia-free type have been in operation at Bergen Park or elsewhere at the times of earlier major earthquakes. Thus we have so far only one case of this kind.

\section{Theoretical models}

There is evidently a close coincidence in time between the seismic $M$ waves and the magnetic variations at Bergen Park. This makes it reasonable to assume that the magnetic waves have their sources fairly close to the observatory rather than, for example, ar the focus of the earthquake.

\subsection{Piezoelectric and piezomagnetic effects.}

In principle the elastic deformations due to seismic waves might give rise to electric and magnetic polarizations (piezoelectrics and piezomagnetics). The strains and shears in the crust are of the order $2 \pi a / \lambda$ ( $a=$ wave amplitude, $\lambda=$ wavelength). For the large surface waves this quantity is about $4 \times 10^{-7}$.

Breiner (1964) has discussed the piezomagnetic effect in a spherical region at the time of a local earthquake. He also gives a number of references to reports of laboratory experiments pertaining to magnetic rocks under stress, e.g. Stacey (1962). On the basis of these results we may try to estimate the magnitude of the piezomagnetic effects associated with the seismic surface waves.

Within certain limits the magnetic susceptibility as well as the remanent magnetization of rocks decrease in the direction of an applied compressive stress (and to a lesser extent increase at right angles to the stress). For a change in stress, $\Delta \sigma$, these relationships can be expressed as

$$
\begin{aligned}
& \Delta \chi=-S_{\chi} \Delta \sigma \chi_{0} \\
& \Delta I=-S_{I} \Delta \sigma I_{0}
\end{aligned}
$$

where $\Delta \chi, \chi_{0}$ and $\Delta I, I_{0}$ are the change and initial values of the susceptibility and remanent magnetization respectively. The strees sensitivities, $S_{\chi}$ and $S_{I}$, are approximately equal and reported to be $0.7-2 \times 10^{-4} \mathrm{~cm}^{2} / \mathrm{kg}$.

For simplicity, let us consider a uniformly magnetized sphere beneath Bergen Park with a radius $r$ of the order of some $\mathrm{km}$. Suppose further that its center is situated at the depth $r$. Its susceptibility $\chi_{o}$ is assumed to be $2 \times 10^{-3}$ e.m.u. This is in accordance with data indicating a magnetic anomaly of some $1000 r$ at Bergen Park.

The value of $\Delta \sigma$ is determined from $\Delta \sigma=E \Delta L / L$ where $E$ is Young's modulus. According to numerical data given by Gutenberg (1959) $E=10^{6} \mathrm{~kg} / \mathrm{cm}^{2}$ is a reasonable value for some common rocks. For the fractional elongation $\Delta L / L$ we may use the value $4 \times 10^{-7}$. This gives 


$$
\Delta \sigma=0.4 \mathrm{~kg} \mathrm{~cm}^{-2}
$$

The change in magnetization of the sphere is given by

$$
\Delta M=\frac{4}{3} \pi r^{3} \Delta \chi
$$

The alternating magnetic field on the surface of the magnetized sphere has the maximum amplitude

$$
\Delta B=\frac{2 \Delta M}{r^{3}}=-2(4 \pi / 3) S_{x} \chi_{0} \Delta \sigma
$$

Inserting $S_{x}=10^{-4} \mathrm{~cm}^{2} \mathrm{~kg}^{-1}, \quad \chi_{0}=2 \times 10^{-3}$ gauss, $\Delta \sigma=0.4 \mathrm{kgcm}^{-2} \quad$ we get

$$
\Delta B=-0.06 \gamma
$$

A similar result would be obtained for a sphere with remanent magnetization. The direction of $A B$ is uncertain.

The observed variations in $F$ are about $0.2 \gamma$. With some adjustments of the parameters used above and a more realistic shape of the magnetized body it does not appear unlikely that piezomagnetism could explain at least part of the observed $\Delta F$.

The pizoelectric effect depends on the reaction of certain crystals to stress. Ih the literature pizoelectrics is discussed for such substances as quartz, tourmaline etc. The strains mentioned above when applied to a quartz crystal would produce electric fields which are very large from a geophysical point of view. Yet, in view of the polycrystalline structure and chemical constitution of the crust it seems unlikely that appreciable net effects could be produced in nature.

\subsection{Magnetic field gradients}

Due to the attenuation of the seismic amplitudes with depth a relative motion exists between a magnetized body in the crust and the magnetic instrument on the surface.

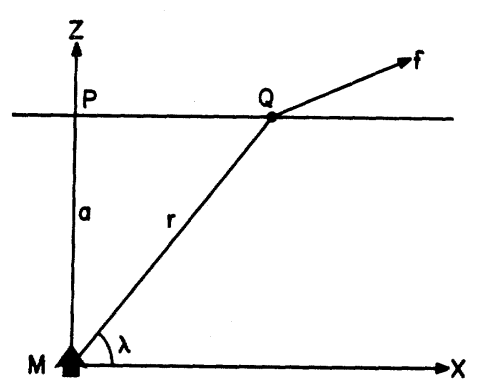

Fig. 9 A magnetic dipole beneath the earth's surface.

For simplicity, let us assume a vertical dipole $M$ buried at a depth $z$ below the surface and producing a disturbance field $f$ on the surface, see Fig. 9. In $P$ we have

$$
f_{p}=\frac{2 M}{z^{3}}
$$

At a point $Q$ on the surface with coordinates $x$ and $z$ the $f$ from the dipole has the components

$$
f_{x}=3 x z \frac{M}{r^{5}}
$$

$$
f_{z}=\left(2 z^{2}-x^{2}\right) \frac{M}{r^{5}}
$$

whence 


$$
f=\sqrt{f_{x}^{2}+f_{z}^{2}}=\frac{M\left(x^{2}+4 z^{2}\right)^{1 / 2}}{\left(x^{2}+z^{2}\right)^{2}}
$$

Now the field gradients $\partial f / \partial x, \partial f / \partial z$ may be determined. It can be shown that

$$
\left|\frac{\partial f}{\partial x}\right|_{\max } \approx \frac{f_{p}}{z} \quad \text { for } \quad x \approx 0.5 z
$$

and

$$
\left|\frac{\partial f}{\partial z}\right|_{\max }=\frac{3 f_{p}}{z} \text { for } \quad x=0
$$

If $\Delta s$ is the displacement of the dipole relatively to a point on the crust, and $\Delta f$ is the amplitude of the observed magnetic variation, we get for the vertical case

$$
\Delta f=\frac{3 f}{z} \Delta s
$$

Let us put $f_{p}=1000 \gamma$. Data indicate that a magnetic anomaly of this magnitude may exist in the Bergen Park area. If we place our dipole at the depth $z=10 \mathrm{~km}$ where $\Delta s$ can certainly not exceed $1 \mathrm{~cm}$ (see Stoneley and Hochstrasser, 1957, and Stoneley, 1958), we get

$$
\Delta f<\frac{3 \times 10^{3} \times 1}{10^{6}}=3 \times 10^{-3} \gamma
$$

This is only about $1: 100$ of the observed amplitude. Similar results are obtained for any reasonable set of values $f_{p}, \Delta s$, and $z$.

The movement of the magnetometer in a field gradient does not appear to be a likely explanation of the observed variation.

\subsection{The induction effect}

\subsubsection{Basic expressions}

The earthquake displacement

$$
s=a \sin \omega t
$$

corresponds to a ground velocity

$$
v=\frac{d s}{d t}=\frac{2 \pi a}{T} \cos \omega t
$$

In a frame of reference moving with the ground we have the electric field

$$
\overline{E^{\prime}}=\bar{E}+\bar{v} \times \bar{F} / c+m \frac{d V}{d t / e}
$$

In the present case the last terim s negligible. $\bar{E}$ is the electric field observed from a frame at rest. If a conductor moves as a whole in a magnetic field, without change of flux, a separation of charges occurs which gives the electric field

$$
\bar{E}=-\bar{v} \times \bar{F} / c
$$


This makes $\bar{E}^{\prime}=0$. One example of this is the earth rotating in its own magnetic field. In the case of earthquakes, however, a deformation takes place and closed current loops are set up, with $\bar{E}^{\prime}$ as the effective electric field. We get the current density

$$
\bar{i}=\sigma(\bar{E}+\bar{v} \times \bar{F} / c)
$$

In some applications the induced currents may be closed through comparatively small resistances. In this case $\bar{E} \approx 0$ so that

$$
\bar{i}=\sigma \bar{v} \times \bar{F} / c
$$

This assumption is not essential for the subsequent discussion. A simple electric circuit with some important features in common with the geophysical case will be discussed in a later section.

\subsubsection{Induced current loops}

In the seismic wave field let us consider one velocity ridge and the adjacent trough, both of width $\lambda / 2$ (one half of a wavelength). The ground velocities have different signs in the two ranges. Neglecting the attenuation of the wave amplitude with distance from focus we find the velocities $\bar{v}$ and $-\bar{v}$ symmetrically spaced about the border line $(\bar{v}=0)$. Specifically, we find the amplitude values $\bar{v}_{O}$ and $-\bar{v}_{O}$ a distance $\lambda / 2$ apart. The same reasoning holds for the induced currents $\bar{i}$. They flow parallel but in opposite directions on the two sides of the borderline. Under certain conditions they form closed circuits. This is illustrated in a qualitative way in Fig. 10 An extreme case would be currents flowing concentrically around the epicenter, without crossing the border-line. The current loops move with the speed of propagation of the earthquake waves.

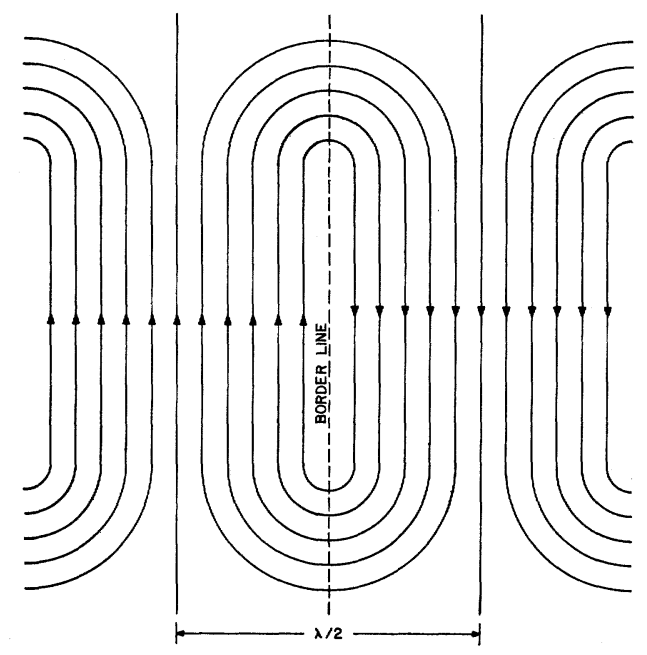

Fig. 10 Seismically induced current loops.

\subsubsection{Calculated electric fields at Bergen Park}

In order to study more closely what ground motions and induced currents can be ex- 


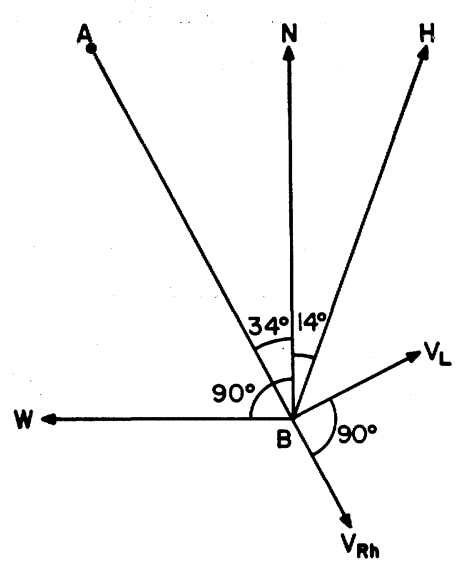

Fg!. 11 The seismomagnetic dircetions at Bergen Park.

pected as an effect of the Alaskan earthquake at Bergen Park, see Fig. 11. A corresponds to the epicenter (Anchorage), $B$ to the recording station at Bergen Park. The line $A B$ which is the direction of propagation of the seismic surface waves, forms an angle of approximately $48^{\circ}$ with the local magnetic meridian in $B$.

During passage of a Rayleigh wave the motion of a surface particle is elliptic and retrograde, the major axis occurring in the vertical direction with about 1.7 the amplitude of the displacement in the direction of propagation. The Love waves are large transverse surface waves.

In the following we use the notations $L=$ Love wave, $R=$ Rayleigh wave, $h=$ horizontal, $v=$ vertical, and $H, D, Z$ for the geomagnetic components. We can now evaluate the components of the electric field according to (15). The vector products of the components of the ground velocity and the field are determined with reference to Fig. 11. The Love velocity and the horizontal and vertical Rayleigh velocities are denoted by $V_{L}, V_{R h}$, and $V_{R v}$, respectively.

In Table 2 we have listed the amplitude values of the electric field components and their directions. The numerical values are based on the magnetic values $H=0.22$ gauss, $Z=0.52$ gauss, and the seismic peak velocities (corresponding to 20 secperiod): $V_{R h}=V_{L}=0.4 \mathrm{~cm}$ $\mathrm{sec}^{-1}, V_{R v}=0.7 \mathrm{~cm} \mathrm{sec}^{-1}$. The latter values are estimates from the Tucson rapid-run magnetograph (used as seismograph), since no useful seismograms were available for the Bergen Park area.

Table 2

\begin{tabular}{l|c|l}
\hline \multicolumn{1}{c|}{ Electric Field } & Numerical value & \multicolumn{1}{c}{ Direction } \\
\hline$E_{R h H} \sim V_{R h} H \sin 48^{\circ}$ & $0.07 \mathrm{mV} / \mathrm{km}$ & vertical \\
$E_{R h Z} \sim V_{R h} Z$ & $0.21 \mathrm{mV} / \mathrm{km}$ & horiz. $\perp A B$ \\
$E_{L H} \sim V_{L} H \sin 42^{\circ}$ & $0.06 \mathrm{mV} / \mathrm{km}$ & vertical \\
$E_{L Z} \sim V_{L} Z$ & $0.21 \mathrm{mV} / \mathrm{km}$ & horiz. along $A B$ \\
$E_{R V H} \sim V_{R V} H$ & $0.15 \mathrm{mV} / \mathrm{km}$ & horiz. $\perp H$ \\
\hline
\end{tabular}

\subsubsection{Observed electric fields}

The values and directions given in Table 2 indicate a maximal horizontal amplitude of about $0.34 \mathrm{mV} / \mathrm{km}$. The values observed at the telluric system of Bergen Park are above $1 \mathrm{mV} / \mathrm{km}$.

There may be some doubt whether a telluric line shunted to the earth and sharing its motion would record the induced electric field. As shown in the next section it is likely that it does.

The 'discrepancy between calculated and observed electric fields may or may not be significant. The ground amplitudes at Bergen Park can in reality be somewhat larger than 
those used here. More important is that the model of the induced telluric currents given here is too simplified. In a more realistic model various potential gradients could add up to the observed value. This will be discussed later.

\subsubsection{Equivalent circuit}

For a survey of the induction currents a simplifield equivalent circuit may be helpful. The circuit depicted in Fig. 12 is in some respects similar to an induced current loop. It consists on $n$ voltaic cells, each with e.m.f. $\varepsilon$ and inner resistance $R_{i}$. The total e.m.f. $n \varepsilon$ corresponds to the induced e.m.f. in the earth. The "outer resistance" of the circuit is $R_{y}$.

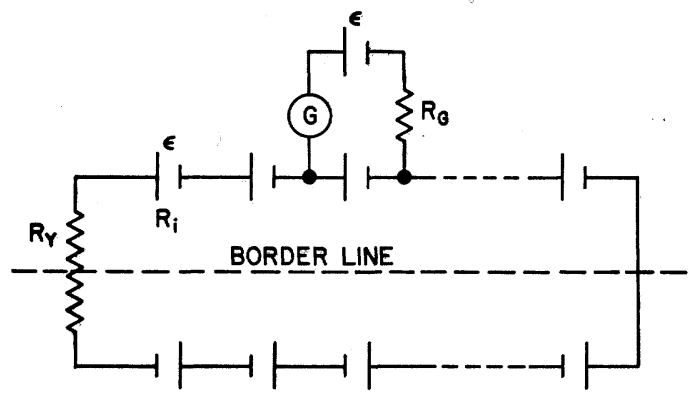

Fig. 12 Equivalent circuit.

Across one of the cells a galvanometer circuit $G$ is connected. This corresponds to the telluric line. Since it takes part in the earth's motion it also contains an e.m.f. $\varepsilon$. Its resistance $R_{g}$ is of the order 1 megohm. Thus $R_{g} \gg n R_{i}+R_{y}$.

Kirchhoff's law gives the e.m.f. $I_{G} R_{G}$ indicated on the galvanometer in the moving frame of reference

$$
\varepsilon_{m}=I_{G} R_{G}=-\frac{\varepsilon n R_{i}}{n R_{i}+R_{y}}
$$

If $n R_{i} \gg R_{y}$ we get

$$
\varepsilon_{m}=-\varepsilon
$$

The telluric recorder thus measures the induced electric field. In a frame at rest we would get the following galvanometer reading

$$
\varepsilon_{r}=\varepsilon-\frac{\varepsilon n R_{i}}{n R_{i}+R_{y}}=\varepsilon+\varepsilon_{m}
$$

with the special cases

$$
\begin{aligned}
& \varepsilon_{r}=0 \text { for } n R_{i} \gg R_{y} \\
& \varepsilon_{r}=\varepsilon \text { for } n R_{i} \ll R_{y}
\end{aligned}
$$

The equation $\varepsilon_{m}=\varepsilon_{r}-\varepsilon$ corresponds in some respects to

$$
\bar{E}^{\prime}=\bar{E}+\bar{v} \times \bar{F} / c
$$

If the galvanometer were instead shunted over a fraction $1 / m$ of $R_{y}$ we would get a reading $\varepsilon_{y}$. Assuming that the telluric line has now a direction perpencicular to its earlier 
direction, it has no e.m.f. $\varepsilon$. We get

$$
\varepsilon_{y}=\frac{R_{y} I}{m}=\frac{n \varepsilon R_{y}}{m\left(n R_{i}+R_{y}\right)}
$$

For $R_{y} \gg n R_{i}$ and for a given length of the telluric line (say $1 \mathrm{~km}$ ) this would correspond to an electric field expression

$$
E_{y}=\frac{n}{m}|\bar{v} \times \bar{F} / c|
$$

Under certain conditions we could easily have

$$
E_{y}>|\bar{v} \times \bar{F} / c|
$$

as indicated on the Bergen Park telluric recorder.

A large metal structure (e.g. an aluminum roof) close to the magnetometer could in itself easily have a conductance comparable with that of a large portion of the crust. Large currents in this structure could modify or cancel the magnetic variations due to ground currents. In the practical case however the connections between the structure and the crust would involve a large resistance. Thus only a negligible fraction of the earth-currents would pass through the metal.

\subsubsection{The telluric recorder}

Some caution may be needed in the quantitative interpretation of telluric data. The standard telluric equipment contains moving parts (e.g. the suspended system of the galvanometers) and may be susceptible to vibrations. One example of this is the earth-current equipment in Niemegk. The recording system at Bergen Park is purely electronic and should be safe in this respect. It did not respond noticeably to the rapid vibrations preceding and following the $M$ waves. As mentioned before suspended magnets responded to the whole spectrum of seismic vibrations. So did the telluric equipment in Niemegk.

During the hours preceding and following the earthquake the two telluric traces (NS and EW) at Bergen Park showed a curious parallelism most of the time. This may indicate some kind of instrumental "cross talk" and tends to cast some doubt on the quantitative reliability of the equipment at the time of the earthquake.

On the other hand: outside the earthquake effect the electric and magnetic registrations at Bergen Park for long-periodic waves appear to be consistent with the main conductivity of the crust, cf Table 3.

The conclusion of the previous discussion is that we may have some confidence that the telluric recordings are quantitatively correct. For complete security independent observations are needed.

\subsubsection{Induced currents in the "normal" crust}

According to Table 2 the two largest electric fields are $E_{R h Z}$ are $E_{L Z}$. They are both horizontal. Only $E_{R h z}$ is parallel to the seismic wave front and thus capable of producing 
current loops of the kind shown in Fig. 10.

The distribution of $i$ within the earth depends on the attenuation of the earthquake amplitude $a$ with depth. The surface waves are guided waves being confined to a channel which is defined by the free surface and the bottom of the crust.

Stoneley and Hochstrasser (1957) and Stoneley (1958) have made theoretical studies of the attenuation of Rayleigh waves and Love waves. They consider a double surface layer of total thickness $s=T_{1}+T_{2}$. If we identify this layer with the earth's crust ( $s \approx 30 \mathrm{~km}$ ) we find that for the large surface waves $(\lambda=3 s)$ the value of $a$ decreases comparatively slowly within the crust. It seems safe, according to this model, to assume an average amplitude of about $0.7 a$ within the uppermost $30 \mathrm{~km}$ of the earth.

To determine the order of magnitude of the induced field the following discussion should be sufficient. We consider an infinitely long slab of the crust directed parallel to the seismic wavefront. Its width is $\lambda / 2$ and its thickness $s$ is the thickness of the crust. For simplicity, we assume that the slab as a whole performs horizontal vibration (perpendicular to its length) of the average amplitude $0.7 a_{R h} / \sqrt{2}=0.5 a_{R h}$. We get the induced current density

$$
i=\frac{\sigma \pi a_{R h} Z}{c T}=i_{0} \cos \omega t
$$

A direct current of density $i_{o}$ would give a magnetic field in the center of the upper side of the slab with the horizontal component

$$
\Delta H=0.4 i_{0} \int_{0}^{s} \int_{0}^{\lambda / 4} \frac{z d z d x}{x^{2}+z^{2}}
$$

Here $z$ is the vertical axis; the $x$-axis is perpendicular to the wavefront. For finite values of $\lambda$ we get

With

$$
\begin{gathered}
\Delta H<0.2 \pi i_{0} s \\
V_{R h}=0.4 \mathrm{~cm} \mathrm{sec}^{-1} ; s=30 \mathrm{~km} ; \\
\sigma=10^{-6} \mathrm{Ohm}^{-1} \mathrm{~cm}^{-1} \text { we get } \\
\Delta H<10^{-4} \gamma
\end{gathered}
$$

The $\sigma$ value is representative for granite and dry sand. It also agrees well with the value obtained for long-period pulsations according to Table 3.

In reality $i$ is an alternating current. The electric field in the crust is determined by the seismic amplitudes rather than by the skin effect. The magnetic variations generated at a certain depth decrease somewhat faster than a magnetostatic field when propagated to the surface. The value of the alternating $\Delta H$ at the surface is therefore certainly not greater than the value corresponding to direct currents.

The observed $\Delta F$ at Bergen Park is $0.2 \gamma . \Delta F$ is of course the resultant field of $\Delta H$ and $\Delta Z$. Since $\Delta Z$ from the slab current is of the same magnitude as $\Delta H$, the observed field is about 1000 times larger than the calculated field. It therefore appears completely unlikely that the vibration of the "normal" crust can explain the observed $\Delta F$.

A small residue of the surface tremor may reach depths of some $700 \mathrm{~km}$ where the mantle conductivity is appreciable. Due to the attenuation of the electromagnetic waves it is unlikely 
that fields from such depths would be noticeable on the surface. According to Gaignard (1956) the "depth of penetration" $\zeta$ where the amplitude is reduced by $1 / e$ is given by

$$
\zeta=\frac{1}{2 \pi} \sqrt{\frac{T}{10 \sigma}} \quad(\text { in } \mathrm{km})
$$

For $T=20 \mathrm{sec}$ and $\sigma=10^{-3} \mathrm{ohm}^{-1} \mathrm{~cm}^{-1}$ (a rough average for the uppermost $700 \mathrm{~km}$ ) we get $\zeta=7.1 \mathrm{~km}$.

\subsubsection{Enhanced conductivity}

To explain the observed $\Delta F$ at Bergen Park as an induction effect we must assume a local conductivity anomaly. If "normal" conductivity conditions had provided an explanation the effect would be universal. As mentioned earlier the effect was not observed at other stations operating inertia-free instruments.

The Bergen Park Observatory is situated in an area rich in minerals. A conductivity anomaly close to the surface would therefore not appear unlikely. It could of course also be located at greater depths where the earth tremor was still appreciable. For example, Rikitake (1964) assumes an anomaly at a depth of at least $100 \mathrm{~km}$ beneath Japan to explain observed magnetic variations.

For plane electromagnetic waves penetrating a homogeneous earth, the following expression may be deduced from Maxwell's equations (Gaignard, 1956)

$$
\rho=\sigma^{-1}=20 T\left(\frac{E_{y}}{H_{x}}\right)^{2} \text { ohm. cm }
$$

$H_{x}$ and $E_{y}$ are the magnetic and electric fields observed on the surface. In homogeneous soil the value of $\rho$ would be independent of $T$. If not, the dependence indicates that $\rho$ as a function of depth is not constant.

In the hours preceding and following the earthquake, pulsations of magnetospheric origin were abundant on the Bergen Park records. The present writer selected four events of different period, avoiding beat-type effects. According to the normal magnetogram of the USC \& GS's Boulder Observatory the pulsation activity was almost completely limited to the $H$ component. Assuming this to be the case also in Bergen Park, $H_{x}$ was derived from the expression

$$
H_{x}=\Delta H=(F / H) \Delta F
$$

As $E_{y}$ the telluric $E W$ component was used. Since the magnetic declination in this area is about $14^{\circ} \mathrm{E}$ no great error is introduced. Average amplitudes over several cycles were scaled.

Table 3 summarizes the results of this investigation. The assumptions of plane waves and a horizontally stratified earth are oversimplifications, especially in a mountainous region as that of Bergen Park. Still, the results may indicate the order of magnitude and tendency of variation of the quantities involved.

If the results in Table 3 are reliable they indicate that with increasing period the resistivity approaches the value $10^{6} \mathrm{ohm} . \mathrm{cm}$ assumed to be valid for the crust. For the more 
Table 3

\begin{tabular}{r|c|c|r|r|l}
\hline Date 1964 & U.T. & $\begin{array}{c}H_{x} \\
\gamma\end{array}$ & $\begin{array}{c}E_{y} \\
m V / \mathrm{km}\end{array}$ & $\begin{array}{c}T \\
\mathrm{sec}\end{array}$ & $\begin{array}{c}\rho \\
\text { ohm. cm }\end{array}$ \\
\hline March 28 & 0300 & 0.17 & 0.9 & 11 & $0.062 \times 10^{5}$ \\
27 & 2325 & 1.50 & 19.5 & 45 & $1.52 \times 10^{5}$ \\
28 & 0757 & 0.73 & 12.2 & 72 & $4.0 \times 10^{5}$ \\
28 & 0834 & 0.87 & 14.7 & 133 & $7.6 \times 10^{5}$ \\
\hline
\end{tabular}

shallow waves of shorter periods, $\rho$ decreases rapidly. This supports the assumption of a high conductivity region in the upper crust.

This is probably not simply a homogeneous layer of enhanced conductivity but a complicated structure with anisotropic properties. All kinds of induced current loops may be set up, horizontal as well as vertical. Since $F$ in Bergen Park has a close resemblance to $Z$ in Tucson (regarded as seismic) vertical movements probaly play an important role:

Wide range induced currents might be closed through narrow horizontal channels close to the surface. Effects of this kind could help to explain the observed value of the electric field at Bergen Park, by adding to the induced field the potential gradients associated with these currents.

The telluric lines have components perpendicular as well as parallel to the seismic wavefronts and should therefore respond to electric fields across these fronts. This would correspond to a galvanometer shunted over part of the resistance $R_{y}$ in the simplified analog circuit considered earlier.

A region with average conductivity $10^{-2} \mathrm{ohm}^{-1} \mathrm{~cm}^{-1}$ would have to be a few $\mathrm{km}$ thick to explain the observed effects. The usual relatively thin top layer of water-impregnated soil and rock alone is not sufficient.

As mentioned before, no noticeable magnetotelluric effect was observed simultaneously with the rapid waves preceding and following the $M$ waves. A few tentative explanations might be suggested here.

(1) The effects are too small to be distinguished from ionospheric and instrumental noise.

(2) Due to the short wavelengths, effects of waves in opposite phases tend to cancel each other.

(3) The periods of the $M$ waves correspond to an electromagnetic pass-band determined by the local conditions.

\section{Conclusion}

Of the mechanisms discussed here piezomagnetic oscillations and induced currents in a region with enhanced conductivity appear most likely to explain the observed electric and magnetic variations at Bergen Park. Clearly, many more observations and a better knowledge of the local elastic, electric, and magnetic properities of the crust are needed for a quantitative treatment. Since the seismic waveform at Bergen Park is not accurately known it is 
difficult to judge which mechanism is most favored by the observed variations. It seems evident, theoretically as well as empirically, that the properties of the "average crust" cannot explain the observed magnetic variations at Bergen Park. This is probably also true for the electric variations.

\section{Acknowledgements}

The present writer is indebted to a number of persons for providing data and comments. Many of them have been mentioned earlier in the paper. I would also like to include Messrs Wallace H. Campbell, Robert Cohen, James R. Heirtzler, and William S. Lee. The valuable help of Mr. W. Paulishak of World Data Center A is hereby acknowledged. In the border area between seismology and geomagnetism discussions with Messrs Markus Båth, Uppsala, and Carl Stepp, U.S.C. \& G.S., proved to be very helpful. Messrs D.G. Knapp, U.S.C. \& G-S., and Hans Voelker, Göttingen, have supplied valuable comments on various parts of the paper.

\section{References}

Anderson, J.A. and H.D. Wood, Journ, Opt, Soc, Amer. 8, 817, 1924.

Båth, M., Seismological Bulletin, March 1-31, 1964, Seismological Institute Uppsala, 1965.

Bauer, L.A., Magnetograph records of earthquakes with special reference to the San Francisco earthquake, April 18, 1906. Terr. Mag. XI, 135-144, 1906.

Breiner, S. Piezomagnetic effect at the time of local earthquakes. Nature, 202, 790-791, May 23, 1964.

Burbank, J.E., Earthquake disturbances recorded on the magnetographs at the observatories of the United States Coast and Geodetic Survey. Terr. Mag. X, 113-125, 1905.

Chapman, S., A note on two apparent large temporary local magnetic disturbances possibly connected with earthquakes, Terr. Mag. 35, 81-83, 1930.

Davison, C., On a possible cause of disturbance of magnetic compass-needles during earthquakes, Geol. Mag. II, 210-211, 1885.

Davison, C.,A study of recent earthquakes, London, 158, 1905.

Gaignard, L., Electricité tellurique, Encyclopedia of Physics, SpringerVerlag, 47, 407-469, 1956.

Gutenberg, B., Physics of the earth's interior, Academic Press, 1959.

Gutenberg, B., and C.F. Richter, Magnitude and energy of earthquakes. Annali di Geofisica, 9, 1-15, 1956.

Liznar, J., Einfluss des Erdbebens vom April 1895 auf die magnetographen in Pola und Wien nebst einigen Bermerkungen über die Wirkung der Erdbeben auf magnetische Variations-apparate überhaupt. Meteor. Zeitschr., XII, 261-267, 1895.

Mascart, M. C.R., CIV, 607-608, 1887.

Moureaux, M. and M. Mascart, Sur les relations qui peuvent exister entre les perturbations magnetiques el les tremblements du 30 Mai 1889. C.R. CVIII, 1189, 1889.

Moureaux, M. and M. Mascart, Sur la cause de certains troubles observés sur les courtes des magnetographes. C.R. CIX, 272-274, 1889.

Reid, H.F., The free and forced vibrations of a suspended magnet, Terr. Mag. XIX, 57-72 and 189-203, 1914.

Rikitake, T., Outline of the anomaly of the geomagnetic variations in Japan, Journal of Geomagnetism and Geoelectricity, XV, 181-184, 1964.

Stacey, F.D., Theory of the magnetic suseptibility of stressed rock, Phil. Mag., 7, 551-556, 1962.

Stonely, R., and U. Hochstrasser, The attenuation of Rayleigh waves with depth in a medium with two surface layers, Mon. Not. Roy. Astr. Soc., Geophys. Suppl. 7, 279-288, 1957. 
Stonely' R., The variation of amplitude and energy with depth in Love waves, Contributions in Geophysics, In Honor of Beno Gutenberg, Pergamon Press, 1958.

Voelker, H., Zur Breitenabhängigkeit erdmagnetisher Pulsationen, Mitteilungen aus dem Max-PlanckInstitut fur Aeronomie, 11, 1963. 